18

\title{
Radiative Properties of a Quantum Dot in a Dielectric Shell*
}

(C) K.K. Pukhov

Prokhorov General Physics Institute of the Russian Academy of Sciences,

119991 Moscow, Russia

e-mail: pukhov@Ist.gpi.ru

Received July 6, 2018

Based on the quantum mechanics and quantum electrodynamics, the general analytical expression is derived for the probability of the spontaneous electric-dipole transition in the semiconductor quantum dot (QD) inside the subwavelength core-shell nanoparticle (NP) embedded in a dielectric medium.

DOI: $10.21883 /$ OS.2018.11.46829.219-18

\footnotetext{
* International Conference „PCNSPA 2018 - Photonic Colloidal Nanostructures: Synthesis, Properties, and Applications“", Saint Petersburg, Russia, June 4-8, 2018.

Полный текст статьи опубликован в английской версии журнала.
} 